\title{
A!
}

This is an electronic reprint of the original article.

This reprint may differ from the original in pagination and typographic detail.

McAvoy, Tom; Jämsä-Jounela, Sirkka-Liisa; Patton, Ron

\section{Milestone report for area 7 industrial applications}

Published in:

Control Engineering Practice

Published: 01/01/2004

Document Version

Peer reviewed version

Please cite the original version:

McAvoy, T., Jämsä-Jounela, S-L., \& Patton, R. (2004). Milestone report for area 7 industrial applications. Control Engineering Practice, 12(1), 113-119.

This material is protected by copyright and other intellectual property rights, and duplication or sale of all or part of any of the repository collections is not permitted, except that material may be duplicated by you for your research use or educational purposes in electronic or print form. You must obtain permission for any other use. Electronic or print copies may not be offered, whether for sale or otherwise to anyone who is not an authorised user. 


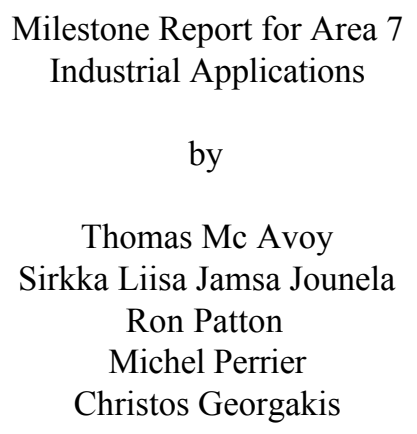

\begin{abstract}
The 2002 IFAC Congress in Barcelona was the first Congress in the new millennium, and thus it offered an unique opportunity for IFAC Technical Committees to plan for their future. Each Coordinating Committee within IFAC was asked to prepare a Milestone Report that summarized past accomplishments and future challenges. The Milestone Report was organized around 6 questions that are given below in the manuscript. For Area 7, industrial applications, the future growth areas that were identified and which are discussed in the paper are: information technology, environmental applications, new sensor technologies, genomics, and mechatronics. Copyright (C) 2000 IFAC
\end{abstract}

Keywords: Industrial applications, chemical, metals, safety, biotechnology, power

Introduction

Coordinating Committee 7 (CC 7), Industrial Applications, consists of four Technical Committees (TC's) organized around control of specific processes, Chemical (CPC), Mining Mineral and $\mathrm{M}$ etals (MMM), Biotechnological (CBP), and Power Plants and Power Systems $\left(\mathrm{P}^{3} \mathrm{~S}\right)$, together with the SAFEPROCESS TC. The methodologies studied under the scope of the SAFEPROCESS TC are applicable to a broader range of areas than the four within Area 7. This report attempts to answer the questions that were posed to CC 7 for developing the B'02 Milestone Report. The questions posed are as follows:

I. What are the most important aspects of your technology?

II. What are the most promising applications of your technology?

III. What are the key control concepts in your technology?
IV. What are the key challenges for your technologies?

V. What are new areas, new applications, new problems, etc.?

VI. Will your technology become more or less important? Why?

The answers to these questions constitute the body of this paper.

Question I. What are the most important aspects of your technology?

Due to the diversity of subjects within $\mathrm{CC} 7$, it is somewhat difficult to give a concise answer to the most important aspects of the technology covered by CC 7 . However, in general profitability is an over-riding aspect of industrial processes, coupled with safe and environmentally friendly operation. Three of the areas within the $\mathrm{CC}$ represent mature technologies, CPC, MMM, and $\mathrm{P}^{3} \mathrm{~S}$. For older processes represented in these areas how to optimize the economics of their operation is often 
the key driver for the application of advanced control. Yet within each area there are new technologies emerging. For example within the chemical area new materials are being developed on a regular basis and the control of these new processes represents a challenging problem. The Biotechnological TC covers a technology that is emerging, and that has enormous potential for mankind in relation to industrially important organisms (e.g. for the production of food products and additives) and biomedically relevant species (e.g. for the production of antibiotics and therapeutics), and to the degradation of pollutants (e.g. wastewater treatment).. Since living organisms are implied, reproducibility of cell cultivations and reduction of variability from run to run deserve special considerations. A more detailed example can be taken from $\mathrm{P}^{3} \mathrm{~S} \mathrm{TC}$. In power plants and power systems the main focus of interest today is the development of controls for the new environment in the power utility industry, which involves restructuring. This environment presents an entirely new challenge since the final shape it will take is still unknown. Therefore although control at the system level will become much more important in the future, it will be an amalgam of some centralized control through the system operators and some local/distributed control as the pattern of generation evolves from the large central generating facilities to independent distributed facilities. The challenge that this area will face involves the co-ordination of controls between various independent entities, each probably having different objectives and goals, to maintain successful and reliable system operation.

Finally, running processes safely, whether they are chemical plants, aircraft, computer systems, transportation systems, etc. is extremely important in today's society. One severe spill from a chemical plant can wipe out years of increased profitability from using advanced control. Detection, diagnosis and isolation of faults before they cause such losses are an important aspect of the work of the SAFEPROCESS TC. Another important technological aspect of the SAFEPROCESS TC is to provide alternative strategies to control the faulty process by using additional (redundant) hardware or software components or relaxing the control performance in order to achieve safe system operation and tolerate some faults (faulttolerant control). Fault detection, diagnosis, isolation and fault-tolerant control are the main functions of a supervisory fault-tolerant process. Safety and reliability are generally achieved by a combination of: fault avoidance, fault removal, fault tolerance, fault detection and diagnosis, and automatic supervision and protection.

Question II. What are the most promising applications of your technology?

This question will be answered individually for each TC.

Chemical Process Control: Model based control is now firmly established in most petroleum refineries the areas represented by CPC. Its use in chemical plants is increasing, but these plants typically are more specialized, have smaller throughputs, and can be more nonlinear than petroleum refineries. All of these aspects are important in terms of how model based control is applied. Chemical companies are currently looking at their entire business operation and the integration of highlevel business models, supply chain management with actual plant operations is becoming more and more important. In the last decade process monitoring and the interaction of process design and control (Process Operability) have emerged as an important application area, and more recently the issue of controller performance monitoring has also come to the forefront. It has been estimated that many single loop process controllers are poorly tuned, and this hurts plant profitability. Similar issues also arise in model based control when a plant changes and the model used for such control needs to be updated.

Metals, Mining, and Minerals: In a recent paper ${ }^{1}$ Prof. Sirkka-Liisa Jamsa Jounela summarized the applications within the MMM TC. She based her sum mary on an examination of the technical meetings sponsored by the TC over the last 20 years. Her results are reproduced in Table 1 below. Only those contributions, which take into account control or monitoring applications with pilot or full-scale industrial processes, were considered. Simulations were not included.

\section{Power Plants and Power Systems:}

The nature of certain traditional functions in the Power Plant and Power System Industry, such as generation scheduling, economic dispatch, etc. will change very significantly because market forces will now govern them. There will be a reversal of the forces driving the operation of the transmission network. Instead of the system operator scheduling generation, 
economic dispatch, etc. throughoptimal load flow, the network will be managed according to market requirements established by contracts of supply and location of load. Further the transmission network will have to be managed by incorporating control devices much more extensively. Such devices include Power Electronic Devices (FACTS), to control the flow of active power and reactive volt-amps over the network while maintaining: angle stability to keep the synchronous system intact, and voltage stability to prevent voltage collapse. Also the integration of distributed generation in the network in the future will become a challenging task.

\section{Control of Biotechnological Processes:}

The control of basic environmental variables like $\mathrm{pH}$, pressure and temperature is well established. The development of soft sensors (based on phenomenological process models or multivariate statistical techniques) to estimate the concentration of substrates, biomass concentration, products and other biologically relevant information such as the state of the fermentation will allow the determination of optimal profiles on-line and the implementation of adaptive nonlinear control techniques to track these profiles. Data mining techniques have shown a great potential to extract information about the state of fermentations and cell cultures that is very difficult to obtain by the usual mechanistic models.

\section{SAFEPROCESS:}

Fault detection and isolation (FDI) and faulttolerant control techniques are being widely incorporated in industrial processes. Important examples where this technology is applicable include continuous complex systems, such as power plants, chemical plants, mineral processing, furnaces, hot/cold metal rolling mills, water-gas-electricity distribution systems, and vehicle/transportation systems (aerospace, automotive systems, surface ships, under water vehicles and railway transportation). In some cases, FDI schemes are being successfully applied in complex components, such as gas and steam turbines, which can be used in vehicles (aircraft, ships, cars) and industrial plants (paper making, plastics, petrochemical, gas industry, combined cycle power generation, etc).

\section{Question III. What are the key control concepts in your technology?}

This question will be answered individually for each TC.
Chemical Process Control: In chemical process control classical control is the most widely used control concept. In addition, almost all control techniques have some applicability in chemical processes. Thus, one finds applications of adaptive control, expert systems, neural networks, etc. However, the most important control methodology that is used is model-based control. Practitioners have turned to using model-based control and optimization to improve plant profitability. Linear model based control is now widely used and can be considered as a mature field. Nonlinear model based control is an important research area. As chemical plants become more agile and they have to respond to a higher frequency of set points changes nonlinear model based control should take on more importance. In addition companies are considering optimizing their entire businesses, and as a result the use of optimization can be expected to increase. The area of supply chain management will take on increasing importance in the future. In the last decade multivariate statistical methods have emerged as an important set of techniques and they have a wide range of applications. These include process monitoring (including batch processes), fault detection, controller performance monitoring and soft sensing among others.

Metals, Mining, and Minerals: The inability of classical control methods to solve all the application problems of interest in the MMM area has led to further developments in control system methodologies and theory. Since 1991 application of AI methods has grown the most. The applications have mainly concerned three AI methods: expert systems, neural networks and fuzzy control. They are used in nearly $40 \%$ of all applications reviewed by Prof. Jamsa Jounela, and therefore represent the most important methods applied in the control and monitoring of MMM processes. During recent years the increasing use of fault diagnosis and process and quality monitoring techniques indicates that control engineering is expanding to include tasks other than control. This expansion represents an emerging area in the automation of the MMM industry.

\section{Power Plants and Power Systems:}

Cconventional control concepts up to now are widely used in the electrical power industry. But there is an emerging need of changing these concepts because of the fact that power system generation and distribution takes place more and 
more under deregulated market conditions. Therefore new control applications like agentbased methods for exploring market and physical system situations will become increasingly necessary. Also increased use of intelligent methods like expert systems, neural networks, fuzzy sets, genetic and evolutionary algorithms, hybrid systems will take place in this industry. Extended control concepts like co-ordinated and adaptive control of power plants and other control devices, e.g. FACTS, DC/AC-converter of wind power parks etc. will substite in the future the conventional control concepts used up to now. The application of online dynamic security assessment systems will become necessary especially in the European electrical system because of the huge extension of the network to the eastern part of Europe in the future. In this context also wide area control using modern IT technologies (Phasor measurements, internet based communication etc.) is under discussion. As a main basis for these new technologies online data acquisition, data exchange and extended data processing will become quite common in this industry.

\section{Control of Biotechnological Processes:}

Most biotechnological processes operate in batch or fed-batch mode. Hence, classical linear control theory is often inadequate for the control of substrate, cell and product concentrations. Adaptive, nonlinear, and multivariable control based on a mathematical model of the process are required as well as the determination of optimal trajectories. The design of controllers based on population balances is attracting a lot of research attention from a systems theoretic point of view. Techniques from pattern recognition and artificial intelligence have proven very useful for fermentation and cell culture monitoring, diagnosis and control.

\section{SAFEPROCESS:}

The key control concepts used by the SAFEPROCESS TC are quantitative and qualitative model-based diagnosis, expert systems for FDI, computational intelligence, signal based diagnosis and robust control design for tolerant-control schemes. Since the mid1990's computational intelligence (AI) methods, such as fuzzy, neural networks, neuro-fuzzy structures, genetic programming, and hybrid quantitative-qualitatives techniques have being incorporated succesfully in FDI schemes for industrial processes.

Question IV. What are the key challenges for your technologies?
For the four application based TC's the key challenge is to contribute positively to process profitability. For mature applications advanced control can do so by decreasing energy use, increasing throughput, reducing off specification products, enabling market processes to function in former monopolies etc. For new applications, e.g. drugs, electronic materials, etc. control can be a key to getting a process up and running. The ultimate challenge for biotechnological processes is the control and regulation of the intracellular cell metabolism. M ethods for the measurement and control of metabolic fluxes have to be developed. For the SAFEPROCESS TC the most important challenge is to maintain safe, economical and reliable plant operation.

Concerning the environment, safe operation of industrial processes will take on increasing importance in the future. As industrial processes increase in complexity, reliability requirements both at component level and system levels become more challenging and difficult to achieve. Traditional diagnosis methods become less capable of ensuring the reliability of such complex systems. An additional challenge is the development of methods that maintain the degree of plant "visibility" required by the human operator. For this reason the SAFEPROCESS $\mathrm{TC}$ is also concerned with issues of "people in control", the psychological and ergonomic factors which ensure that the human operator can perform efficiently under faulty situations. Developments seek to avoid the undesirable behavior of plant operators in the "3-Mile Island" type of emergency scenario.

\section{Question V. What are new areas, new applications, new problems, etc.?}

The areas, applications, and problems are described individually.

1. Information Technology: Today's world is changing rapidly as companies adjust to global economic competition. Information technology (IT) is currently the main mechanism for streamlining business activities, and is now used widely by companies seeking to improve competitiveness. Technological change and organisational restructuring have been found to bring gains in productivity and market share. Current business is undergoing a major paradigm shift, moving from traditional management into a world of agile organisations and processes. An agile corporation should be able to respond rapidly to market changes. Corporations have been developing a number of 
information technology (IT) systems to assist with various aspects of the management of online business processes. The global market generates a need for global technical support. Telecommunication technology is providing a large number of opportunities for enhancing the speed and quality of the support process by enabling remote access to equipment. A technician can contact equipment located far away from his office, diagnose the equipment status, and suggest appropriate repairs online. In some cases they can even intervene directly in the operation. This remote operation represents considerable time saving and a reduction in the costs of the technical support process.

\section{Environmental Monitoring and Emission}

Control: Environmental control is nowadays an important issue for the survival of the process industries. The monitoring of industrial emissions is the first task in pollution control.

Reduction of industrial wastes such as waste gas and waste materials are an essential measure for environmental protection. Yield improvements and energy savings are also important for reduction in industrial waste, as well as for improving the profit margin of products. Exact process control is necessary for the better yield and reduction of power consumption. Forecasting of environmental conditions around an industrial area is also important for better pollution control. Pressure for environmental protection is driving the process industries to use better, more advanced methods of control and monitoring. In the chemical area plants are being designed for minimum effluents, and such plants will be challenging to control and operate. In the MMM area recycling is a new field that has emerged. An important issue involves how to design and control recycle operations so that the environment is preserved and plants remain profitable. Early detection and isolation of abnormal situations, such as environmental pollution (e.g. in rivers, irrigation systems, wastewater treatment plants,..) has become a key new area for application of FDI methods developed under the remit of the TC SAFEPROCESS.

\section{New Sensor Technologies: In the majority}

of process plants the sensors used for control have been around for many years. These sensors include flow meters, pressure sensors, level sensors, etc. They are robust and they have stood the test of time. However, in most cases these sensors do not measure variables that are key indicators of product quality, which is a key variable that determines process profitability.
Research on modern sensors holds the promise to have a dramatic impact on how process plants are operated. For example, if one could measure chemical composition on line using an inexpensive and robust sensor then problems could be corrected quickly and faster than if one had to wait for a laboratory assay. M icrochemical sensors that are chip based offer one approach to such online chemical sensing. Due to their low cost, these chemical sensors could also be used in environment applications as well. For biotechnological applications, sensors based on image analysis will be useful to characterise the cell population during culture. Similarly, in the MMM area new sensors for measuring particle size distribution, froth characteristics, etc. hold great promise for future application. Research into model-based FDI methods can be seen as a complementary technology ("software" sensors) of process engineering when the hardware sensors malfunction. Sometimes, the model outputs can replace the faulty or unhealthy sensors in a fault-tolerant control scheme. The concept of the software sensor or software actuator is now a part of the development of so-called intelligent actuators or sensors, in which these components become more tolerant to faults, thereby guaranteeing improved plant reliability.

\section{Genomics, Proteomics, Bioinformatics,} Metabolic Engineering: Given the recent development of genomics and proteomics, computational methods need to be developed to integrate and quantify biological data. This information has the potential of elucidating gene regulation mechanisms and identifying genes implicated in disease, or perfecting industrially important high performance strains. Concepts and methodologies of metabolic engineering will have a major impact on the integrated design and control of cellular systems. These methods will allow the systematic study of cellular responses to genetic and environmental perturbations.

5. Mechatronics: Mechanical systems such as robots, car engines etc, are being developed which have an increasing level of integration with sensors, actuators, microelectronics and information processing. They develop an advanced concept, the mechatronics system, characterized through the integration of components (hardware) and signal and modelbased functions (software), resulting in automatic functionality. This is an important area for the SAFEPROCESS TC as all aspects of reliability and safety must be considered as 
key requirements during design, testing, manufacturing and operation. In general, faulttolerance methods must be implemented at component and unit level to improve the reliability and safety of mechatronic systems. Unavoidable faults must be covered by maintenance and on-line supervision, including all the main tasks of the SAFEPROCESS TC: fault-tolerance, protection and supervision with FDI, fault diagnosis and appropriate safety actions.

Question VI. Will your technology become more or less important? Why?

The fields covered by Area 7 will definitely become more important in the future. It is difficult to imagine how chemicals, metals, and power will not grow in the future. The area of biotechnology will almost certainly lead to many significant improvements in society. The rapid changes in biological research are transforming traditional approaches and are generating numerous new opportunities in biotechnology and biomedical research. The new knowledge generated will eventually lead to introducing control at the genetic level. The performance of dynamic control systems is not only being defined when all components work correctly, but also when there is an abnormal situation (a fault in a sensor, actuator, controller or plant component). The concept of degraded but faulttolerant performance of the faulty process is a guarantee that the plant maintains "safe process" operation. Consequently, the tasks of the SAFEPROCESS TC will increase in importance.

Literature Cited:

[1] Jämsä-Jounela, S-L, “Current Status and Future Trends in the Automation of Mineral and $M$ etal Processing", preprints of IFAC Workshop Future Trends in Automation of Mineral and $\mathrm{M}$ etal Processing, Finland, pp. 96-108, Aug. 22-24, 2000.

Table 1: Distribution of the different control methods according to the kind of process

\begin{tabular}{|c|c|c|c|c|c|c|c|}
\hline & $\begin{array}{l}\text { Mineral } \\
\text { process-in }\end{array}$ & $\begin{array}{l}\text { Hydromet } \\
\text { g-allurgy }\end{array}$ & $\begin{array}{l}\text { Electro- } \\
\text { refining }\end{array}$ & $\begin{array}{l}\text { Blast } \\
\text { furnace } \& \\
\text { furnaces }\end{array}$ & $\begin{array}{l}\text { Steel } \\
\text { making \& } \\
\text { continuous } \\
\text { casting }\end{array}$ & $\begin{array}{l}\text { Hot } \\
\text { Roll- } \\
\text { ing }\end{array}$ & $\begin{array}{l}\text { Cold } \\
\text { Roll- } \\
\text { ing }\end{array}$ \\
\hline $\begin{array}{l}\text { Classical control } \\
\text { methods }\end{array}$ & 6 & 1 & 0 & 1 & 0 & 5 & 1 \\
\hline $\begin{array}{l}\text { Model based and } \\
\text { MPC }\end{array}$ & 3 & 0 & 0 & 10 & 0 & 6 & 2 \\
\hline $\begin{array}{l}\text { Multivariable } \\
\text { control }\end{array}$ & 10 & 0 & 0 & 1 & 0 & 2 & 0 \\
\hline Adaptive control & 7 & 0 & 1 & 3 & 6 & 1 & 5 \\
\hline AI & 11 & 1 & 0 & 12 & 10 & 2 & 2 \\
\hline $\begin{array}{l}\text { Fault diagnosis } \\
\text { and Process } \\
\text { Monitoring } \\
\end{array}$ & 8 & 1 & 1 & 9 & 3 & 5 & 0 \\
\hline $\begin{array}{l}\text { Quality } \\
\text { monitoring } \\
\end{array}$ & 2 & 0 & 0 & 1 & 5 & 1 & 0 \\
\hline Others & 0 & 0 & 0 & 0 & 0 & 3 & 0 \\
\hline Total & 47 & 3 & 2 & 37 & 24 & 25 & 10 \\
\hline
\end{tabular}

